\title{
FIRST RESULTS OF USING THE SECOND GENERATION SBAS IN AUSTRALian URBAN AND SUbURBan ROAD ENVIRONMENTS
}

\author{
Ahmed El-Mowafy ${ }^{1}$, Norman Cheung ${ }^{2}$, Eldar Rubinov ${ }^{3}$ \\ 1. School of Earth and Spatial Science, Curtin University, Perth, Australia \\ 2. HMI for Transport for New South Wales, Sydney, Australia \\ 3. FrontierSI, Melbourne, Australia
}

\begin{abstract}
In this study, the first results of the next-generation dual-frequency multi-constellation SBAS-based kinematic positioning in Australian urban environments are presented and analysed. As the standalone GNSS positioning is unable to deliver the accuracy required for absolute positioning in Intelligent Transport Systems (ITS), more advanced technologies are needed, and the Australian SBAS with PPP capabilities is a candidate. Kinematic tests were run in scenarios characterised by four environments: high-density urban, low-density urban, suburban and tree-canopy. SBAS positioning performance was evaluated in the different environments, with a focus on its capability to provide lane identification and thus aid ITS applications.
\end{abstract}

\section{INTRODUCTION}

Connected and automated vehicles (CAV) hold the premise of a safer and more efficient road transport network. However, accurate positioning information is essential. Trials of connected vehicles have shown that Standard Point Positioning (SPP), as it performs in Australia, is not accurate enough to enable many of the cooperative vehicle safety applications envisaged by vehicle manufacturers and road safety researchers (Austroads, 2015). The use of augmentation service and multi-constellation satellite systems, for instance through the use of Satellite Based Augmentation System (SBAS) service, can offer the potential for improving the positioning accuracy and enabling the envisaged applications (TIC, 2012; Austroads, 2015a).

In preparation for building state of the art SBAS in the region, Geoscience Australia (GA), Land Information New Zealand (LINZ) and FrontierSI (previously Cooperative Research Centre for Spatial Information) along with three service providers - Lockheed Martin, GMV and Inmarsat, ran a two year SBAS test-bed to evaluate the performance of SBAS (www.ga.gov.au/scientific-topics/positioning-navigation/positioning-for-the-future/satellitebased-augmentation-system). The trial aimed at testing the proposed SBAS services across ten key industry sectors including aviation, agriculture, resources, road, rail, maritime, spatial, construction, utilities and consumer.

The Australia-New Zealand (NZ) SBAS test-bed has provided three levels of service. The first service was the single-frequency L1 GPS-only SBAS service that is currently used by the various providers around the world (NSTB/WAAS T\&E Team, 2015). This service is able to achieve sub-metre positioning in real-time. The second service was the Dual-Frequency MultiConstellation (DFMC) SBAS service. This service used L1 and L5 GPS and Galileo measurements. The DFMC was expected to provide improvements to the L1 SBAS by using more satellites as well as providing enhanced mitigation of ionospheric errors through the use 
of L1/L5 ionosphere-free combination for GPS, and E1/E5a for Galileo. Except for the Japanese Multi-functional Satellite Augmentation System (MSAS), no other SBAS in the world is currently offering the DFMC SBAS option. The advantages of the ionosphere-free DFMC SBAS include that there is no need for estimation or interpolation of the ionosphere, which gives a better performance during periods of high fluctuations activity of the ionosphere. It also allows for covering a larger area of service than L1 SBAS, for instance DFMC SBAS can cover the whole Australia-Asia Pacific region, and thus is useful for off-shore maritime applications, whereas traditional L1 SBAS is only limited to Australia/NZ region. However, the use of DFMC SBAS requires the more expensive DFMC receivers.

Finally, the third service was the Precise Point Positioning (PPP) service. PPP is a phase-based positioning technique that relies on the use of precise orbit and clock corrections, typically computed from a global network of reference stations. These corrections are transmitted to user to allow them to compute positions accurate to 5-10cm (Zumberge et al., 1997). Whilst the PPP positioning method is superior to L1 and DFMC SBAS in terms of accuracy, it requires a period of convergence time to achieve this level of accuracy, which can be in the range of 30-60 minutes (El-Mowafy, 2018), whereas SBAS L1 and DFMC positioning is achieved instantaneously due to being code-based.

This paper shows the first results in terms of the overall positioning performance of SBAS (L1, DFMC) and PPP positioning methods offered as part of the SBAS test-bed. The main focus is set on its use for advanced transportation applications. Two test schemes were conducted in different scenarios: the first scheme is road safety and the second scheme is heavy vehicle efficiency. The study assessed whether better vehicle positioning through the use of SBAS can be achieved compared with the current approaches that mostly utilise single point positioning. The outcomes of this research can assist in clarifying the potential of different services of SBAS in improving positioning performance and in turn the safety and efficiency of the road transport in Australia. Initial testing shows that performance in the transportation sector is very dependent on the environmental surroundings of where SBAS is being used. Therefore, the analysis is conducted classifying the work environment into four classes: high-density urban, low-density urban, suburban and in the presence of tree canopy. Performance metrics such as the obtained precision, accuracy, availability and suitability of positioning solutions for the studied schemes is discussed.

\section{POSITIONING TECHNIQUES}

SBAS and PPP positioning techniques are summarised in the following sections including signal structure, achievable accuracies and other performance indicators.

\subsection{SBAS}

Any SBAS system is constituted by a ground segment, i.e. a network of reference stations and master stations, and a space segment, i.e. a set of geostationary (GEO) satellites (Enge et al., 1996; Roturier et al., 2001). The SBAS system augments the GNSS systems in three ways:

- by providing measurement differential corrections to improve the positioning accuracy,

- by providing extra ranging navigation signals, from the GEO satellites, and;

- by providing the integrity service, i.e. provide a measure of the trust that can be placed 
in the correctness of the information supplied by the system. The SBAS integrity concept is revolved around civil aviation requirements and might not be applicable to other applications, such as transport. In this integrity service, the system checks presence of faults in satellites and inform users about faulty satellites, which are deemed unhealthy, and provide parameters that facilitate the computation of the Protection Levels (PL) used in integrity monitoring.

The Australian-NZ test-bed did not enable the latter two functions.

The measurements taken at the ground reference stations allow the computation of the satellite orbit and clock and ionosphere corrections. The satellite corrections are common for both the reference stations and the user, therefore they can be applied at the user's location. The ionospheric delay computed at the reference stations is typically interpolated in a grid of $5^{\circ}$ (approximately $500 \mathrm{~km}$, which is also applied in the Australian-NZ SBAS testbed). The user interpolates these grid values at their location, and computes the slant ionosphere by using a mapping function. The general GNSS code and phase observations can be expressed as:

$$
\begin{aligned}
& P_{j}=\left\|x_{s}-x_{r}\right\|+c \delta t_{r}-c \delta t_{s}+l_{j}+\tau+m+c d_{r}+c d_{s}+e \\
& \varphi_{j}=\left\|x_{s}-x_{r}\right\|+c \delta t_{r}-c \delta t_{s}-l_{j}+\tau+\lambda_{j} a+\mu+c \delta_{r}+c \delta_{s}+\varepsilon
\end{aligned}
$$

where $p_{j}$ and $\varphi_{j}$ are the code and phase observables on the $j$ th frequency (in metres), $x_{s}$ and $x_{r}$ are the positions of satellite and user receiver respectively, $c$ is the speed of light in vacuum, $\delta t_{r}$ and $\delta t_{s}$ are receiver and satellite clock offsets correspondingly, $l_{j}$ is the slant ionospheric delay, $\tau$ is the slant tropospheric delay, $m$ and $\mu$ denote the multipath error on code and phase measurements respectively, $d_{r}, d_{s}$ and $\delta_{r}, \delta_{s}$ are systematic hardware delays, at receiver and satellite, on code and phase measurements respectively, $\lambda_{j}$ is the $j$ th carrier wavelength, $a$ is the carrier phase integer ambiguity in cycles, $e$ and $\varepsilon$ are the random code and phase measurements noise, respectively. $x_{r}$ and $c \delta t_{r}$ are the unknowns to be solved for. The other terms are sources of error that need to be accounted for to achieve precise positioning.

SBAS provides corrections for the clock and orbit errors, and the ionospheric delay. Typically, SBAS positioning depends on processing code observations, although in most applications both the user and reference stations employ carrier-phase observation smoothing to code observations to reduce the effect of noise in code observations (Enge et al., 1996). The reference stations make use of geodetic multi-frequency receivers to estimate the ionospheric delay and increase the accuracy of estimating the network corrections. As mentioned earlier, in addition to the legacy L1 SBAS, the Australian-NZ SBAS test-bed included L1/L2 dualfrequency observations for GPS and E1/E5a for Galileo. In a future operational SBAS system, the DFMC will use L1/L5, but since there is currently a limited number of GPS satellites transmitting on the L5 frequency, the decision was made to use L1/L2 combination during the test-bed period to make use of all available satellites.

In SBAS, the total pseudorange variance is generally computed as (e.g. in the WAAS and EGNOS systems, see Walter et al., 2012):

$$
\sigma_{\text {code }}^{2}=\sigma_{U D R E}^{2}+\sigma_{\text {GIVE }}^{2}+\sigma_{\text {tropo }}^{2}+\sigma_{\text {multi }}^{2}+\sigma_{\text {noise }}^{2}
$$

where $\sigma_{U D R E}$ is the standard deviation (STD) of the User Differential Range Error (UDRE), $\sigma_{\text {GIVE }}$ is the STD of the Grid Ionospheric Vertical Error (GIVE), $\sigma_{\text {tropo }}$ is the STD of the 
differential tropospheric delay, $\sigma_{m u l t i}$ is the STD of the (differential) multipath error, and $\sigma_{\text {noise }}$ is the STD of the combined corrections-user thermal noise. The UDRE includes satellite clock and ephemeris residual errors, and satellite hardware delays. In integrity monitoring, satellite and receiver hardware delays are treated separately and added as nominal biases in the computation of the Protection Levels (Blanch et al., 2015). Table 1 provides common values of the different error components in (3) for GPS as an example.

Table 1: Example of typical SBAS range accuracy components (Walter et al., 2012).

\begin{tabular}{|c|c|c|}
\hline Source & L1 & $\begin{array}{c}\text { L1/L5 iono-free comb. } \\
\text { (DFMC) }\end{array}$ \\
\hline$\sigma_{U D R E}$ & $0.3-0.6 \mathrm{~m}$ & $0.3-0.6 \mathrm{~m}$ \\
\hline$\sigma_{\text {GIVE }}$ & $0.2-0.8 \mathrm{~m}$ & $<0.1 \mathrm{~m}$ \\
\hline$\sigma_{\text {tropo }}$ & $0.05-0.3 \mathrm{~m}$ & $0.05-0.3 \mathrm{~m}$ \\
\hline$\sigma_{\text {noise }}$ & $0.2-0.6 \mathrm{~m}$ & $2.6 \times \sigma_{\text {noise } L 1}$ \\
\hline
\end{tabular}

In Table 1, the single-frequency (L1) SBAS service and DFMC (L1/L5) SBAS are distinguished since the latter makes use of the ionosphere-free combination. DFMC SBAS permits the cancellation of the first order error term of the ionospheric delay, which constitutes over $99 \%$ of the ionosphere effect. However, when using ionosphere-free model, the thermal noise, in addition to the multipath error (when treated as a random variable), will be inflated by the factor 2.6. It is expected that in the aviation sector the benefit of elimination of the ionospheric threat will outweigh the increase of noise and multipath errors, and the ionospherefree combination will be employed without the aid of an ionosphere model. However, in Intelligent Transport Systems (ITS), which experience various land-based environments, multipath effects constitutes the most critical error component. Therefore, in this first study, focusing mainly on urban and suburban environment, performance of L1 and DFMC SBAS are compared. Results for the two positioning methods, as shown later, indicate similar performances, with a slight improvement using DFMC. This suggests that the two effects, the reduced ionosphere delay and increased noise/multipath for the DFMC mode, tend to balance out in these environments. However, as stated earlier, the use of DFMC ionosphere-free measurements is advantageous in case of experiencing sudden or large fluctuations in the ionosphere and it is available over a larger area of service.

Performance analysis reports, e.g. by the NSTB/WAAS T\&E Team (2015), show results of WAAS performance over a long period of time. Range error variance smaller than $1 \mathrm{~m}$, positioning horizontal accuracy $0.5 \mathrm{~m}$ to $1.4 \mathrm{~m}$, and vertical accuracy of $0.8 \mathrm{~m}$ to $1.7 \mathrm{~m}$ were reported. The improvement in accuracy, compared to SPP, was generally over 33\% (Ali et al., 2012). However these WAAS services are meant for use in the aviation sector. The multipath term is coupled with the receiver noise and assumed to be of the order of $0.3-0.5 \mathrm{~m}$. This is of course not representative of a typical urban environment, where multipath and no line-of-sight (NLOS) effects can generate errors of several metres (Ali et al., 2012). Moreover, for use in ITS, the SBAS positioning augmentation does not have to comply to the strict regulation in place for aviation applications (Choy et al., 2017), and can make use of not monitored SBAS measurements (for which the integrity information is not available). As a result, SBAS modes designed for ITS can make use of a larger number of observations and show higher availability than aviation modes.

J. of Spatial Science 2019 doi/full/10.1080/14498596.2019.16649434 


\subsection{PPP}

PPP is also an augmentation-based positioning method, but relies on carrier-phase observations to achieve better positioning accuracy than SBAS. Zumberge et al. (1997) introduced the PPP technique in 1997, as a post-processing technique. As precise products with smaller latencies became available, the focus of PPP research shifted to real-time PPP processing (Kechine et al., 2003; Heroux et al., 2004; Gao and Chen, 2004, El-Mowafy, 2017) and, more recently, towards multi-constellation PPP (Melgard et al., 2010; Tegedor et al., 2015).

\section{SBAS HARDWARE USED IN THE TEST}

In this study, both SBAS (L1, DFMC) and PPP performances were evaluated. Whilst many off-the-shelf receivers can pick up L1 SBAS, special hardware and software were required to decode DFMC and PPP messages. A Septentrio AsteRx-U receiver with Leica AR10 antenna, an RF front-end and Linux tablets were used to capture real-time signals and log data for postprocessing. Software packages magicGEMINI and magicAPK, developed by GMV, were employed for post-processing SBAS data and decode real-time PPP messages, respectively. magicGEMINI is a software package running on a Windows platform. It accepts raw GNSS observations as well as SBAS messages and outputs SBAS derived positions and corresponding evaluation of the system performance at the user level. magicAPK runs on a Linux platform and provides PPP solution in real-time. Figure 1 shows a diagram of the hardware and software setup used.

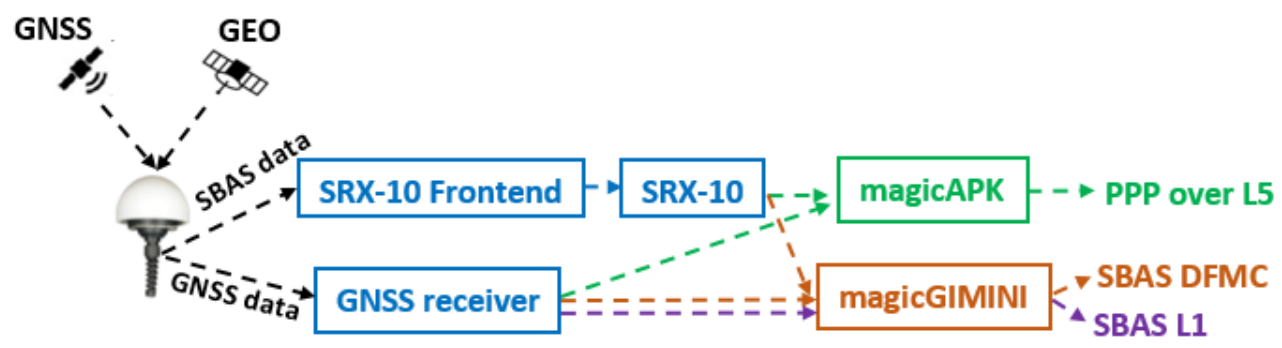

Fig 1: Hardware and software setup used

\section{TESTING AND METHODOLOGY}

\subsection{Test description}

Testing of the second generation SBAS in this study was separated based on two focus areas:

i. Road Safety (also referred to as Test Scheme 1). The test was conducted in Wollongong, Australia, to examine the ability of SBAS in supporting issuing collision alerts to drivers. The positioning requirement for this application is discussed in section 5.6. Two cases were considered:

1. Positioning for forward collision warning, with the following scenarios:

1.1. Two vehicles travelling in the same lane.

1.2. Vehicles travelling in adjacent lanes moving in the same and opposite directions. 
2. Positioning for intersection collision warning, with the following scenarios:

2.1. A stationary vehicle at an intersection with the other vehicle approaching.

2.2. A vehicle travelling on an overpass and the other vehicle travelling underneath it.

These tests were conducted in selected routes in Wollongong, Australia in areas mostly characterised by a suburban environment, with generally good satellite visibility. Exception was for one test run characterised by presence of trees on the sides of the road. Two SBAS kits were mounted on two different test Vehicles, shown in Figure 2. The first two tests were conducted on 1/6/2018 and the latter two tests were carried out on 31/05/2018.

ii. Heavy Vehicle Efficiency (also referred to as Test Scheme 2). The test was conducted in Sydney, Australia, to examine the SBAS-based positioning ability to improve transport network efficiency by identifying which lane the vehicle is travelling in. This facilitates applications such as traffic light signal priority, where the green light is extended for approaching trucks and buses travelling in specific lanes. Two cases were considered:

3. Tracking a vehicle along a bus route

4. Tracking a vehicle in dense urban environment, such as Sydney Central Business District (CBD)

The vehicles used in these tests are shown in Figure 2, with the SBAS antennae mounted on the roof.

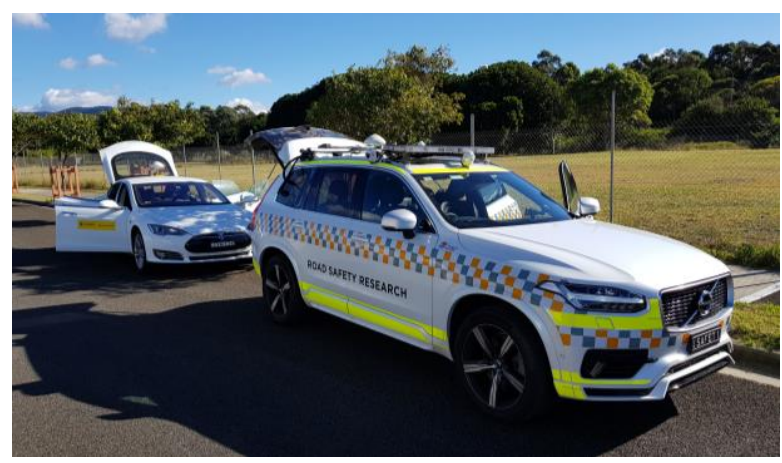

Figure 2: Test vehicles

As mentioned earlier, it was observed that both SBAS and PPP positioning performance is heavily dependent on the environment of application, which varies considerably in the transport sector. Therefore, the environment of the tests was subdivided into the following four categories based on the level of sky visibility (with example shown in Figure ):

1. Suburban: characterised by presence of low-rise buildings, with a maximum of three floors

2. Low-density urban: with the presence of low-rise building and some high-rise buildings on one side of the road

3. High-density urban: characterised by high-rise buildings on most sides of the road

4. Tree canopy: with tree covering most of the road on both sides, sometimes forming tree canopy

The tests of the Heavy Vehicle Efficiency (Test Scheme 2) were carried out in dense urban environment. For the tests in the CBD area, a distinction was made between low-density and high-density urban environments. The bus route was divided into three settings - high-density urban, low-density urban and suburban environments as illustrated in Figure 4. 

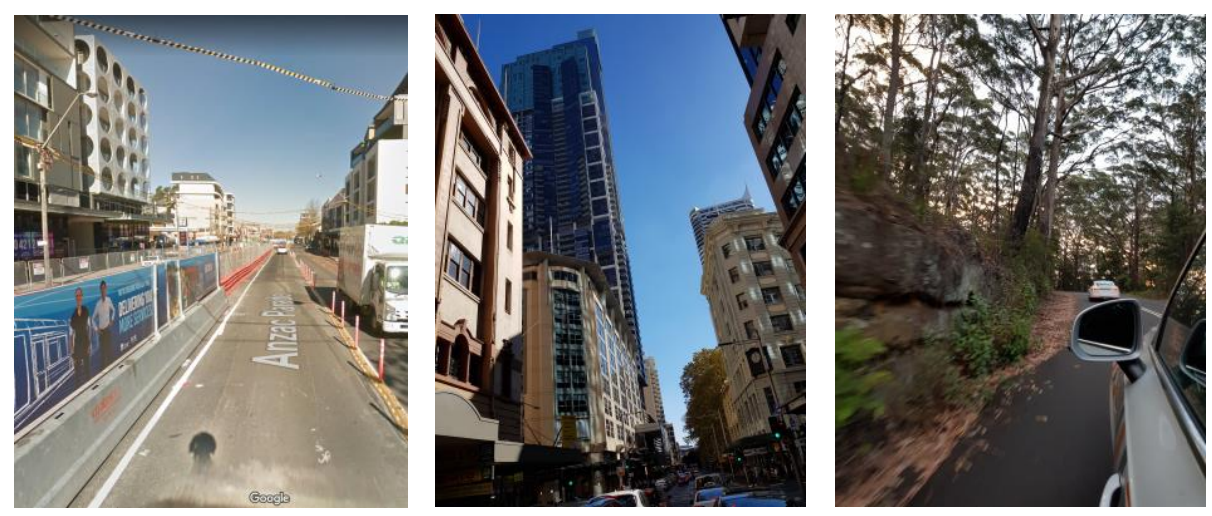

Figure 3: Examples of test environments: from left to right, low-density urban, high-density urban and tree canopy

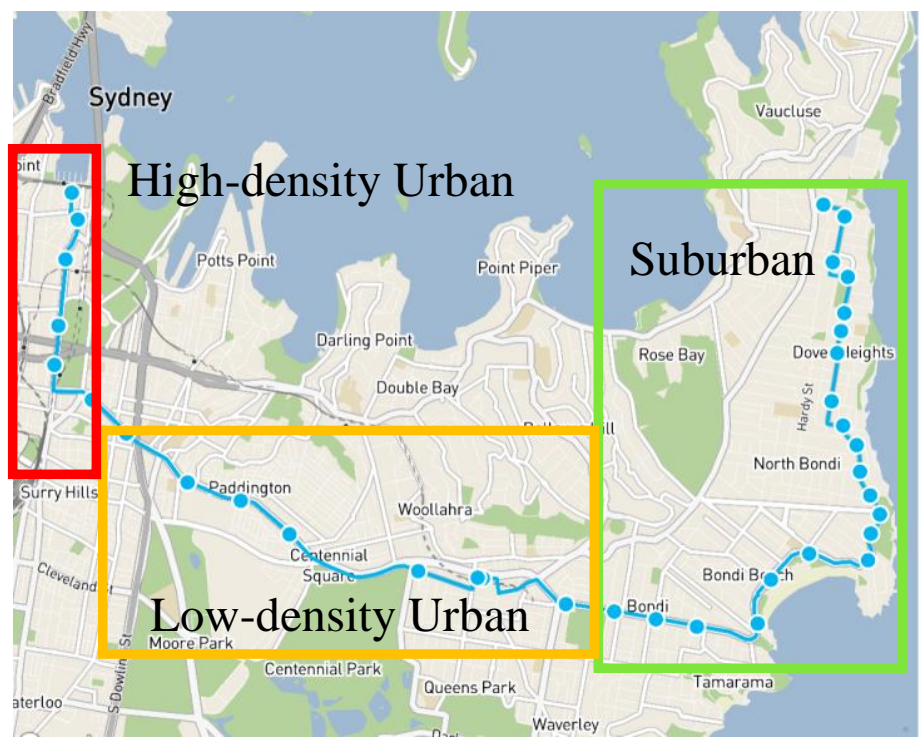

Figure 4: Bus route with subdivision in environments

\subsection{Data analysis methodology}

To evaluate the quality of L1 and DFMC SBAS and PPP positioning results it was necessary to have a reference ground truth for the positions of the vehicle, to which the SBAS and PPP position solutions were compared. This 'ground truth' was computed in post-processing mode through independent relative kinematic positioning (PPK). The rover data comprised the same raw code and phase observations used for SBAS-based positioning. The rover raw observations were stored in the receivers, later downloaded in the office and post-processed relative to a base/reference station. The Continuously Operating Reference Station (CORS) station UNW2 at the University of New South Wales (UNSW) was used as the reference station for the tests carried out in Sydney (Test Scheme 2). The CORS station GONG was used in Wollongong (Test Scheme 1). The data were retrieved through the HxGN SmartNet online service (https://hxgnsmartnet.com/en-AU). In both test schemes, the locations of the rover receivers that were mounted on the test vehicles were always within a radius of eight kilometres from the reference stations. Only the ambiguity-fixed solutions from PPK, which typically provide $1-5 \mathrm{~cm}$ precision, were used as a reliable source for ground truth. 


\subsection{Positioning performance indicators}

The main performance indicators computed in this study to evaluate the quality of SBAS positioning are availability, outlier rate, mean error and root mean square error (RMSE), noting the following:

- Availability is defined by the fraction of time in which a position solution was delivered by the software over the total time of operations.

- Mean Error and RMSE were computed after all outliers were removed by the set of solutions. The mean error is a measure of any bias possibly affecting positioning, whereas the RMSE is a measure of the positioning accuracy.

- The outliers were identified and removed in two steps:

i. All evident outliers were removed, i.e. all solutions with errors larger than 10 metres in case of SBAS and 5 metres in case of PPP.

ii. Next, a preliminary standard deviation of the errors was estimated and all the solutions with errors larger than three standard deviations (corresponding to $99.7 \%$ confidence level) were removed.

\section{POSITIONING RESULTS}

The following sections show representative examples of the test runs conducted in different environments. Since in this study the focus is on some advanced transport applications, where horizontal positioning is of interest, but not vertical positing, only results of the former are presented. The time series of the horizontal positioning errors in North and East directions, the Horizontal Dilution of Precision (HDOP) and the number of measurements (satellites) available at each epoch are shown for the different SBAS positioning modes. The HDOP represents the ratio between the precision of the horizontal solution and the average precision of the range measurements. All data collected and results are plotted at a frequency of $1 \mathrm{~Hz}$.

\subsection{Results of suburban environment}

Figure 5 shows the test trajectory for one day of testing in Wollongong, in test scheme 1, which is a good example of suburban area with good satellite visibility, except for some road stretches that are flanked by trees (shown by red stretches of the track in the Figure ). Figure 6 to Figure 8 depict the North and East positioning errors for one vehicle in this suburban environment as an example. The figures show results for SBAS L1, DFMC and PPP. The gaps seen in some of the figures for some positioning modes are due to the limited number of available observations, for instance due to signal obstruction by structures or trees. The graphs also show the number of raw and corrected measurements. The raw measurements are the measurements available at each epoch, whereas the corrected measurements are those for which an SBAS correction is available, and that are used for positioning.

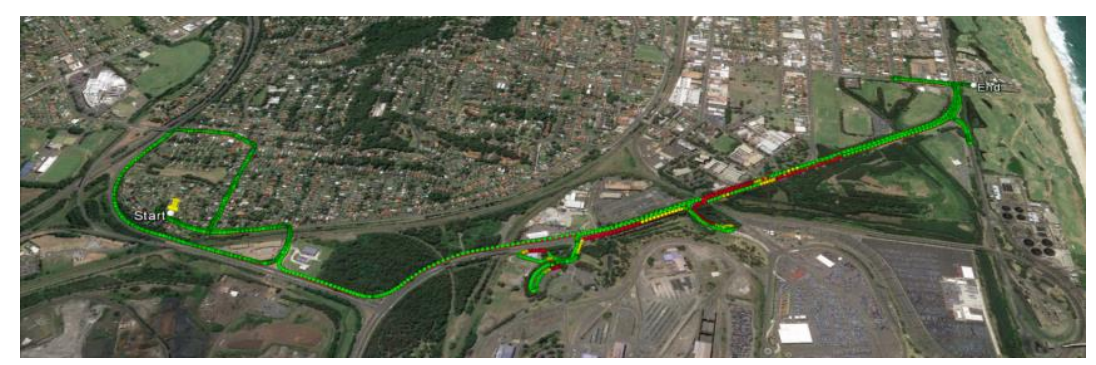

Figure 5: Tracking test in the suburban environment 

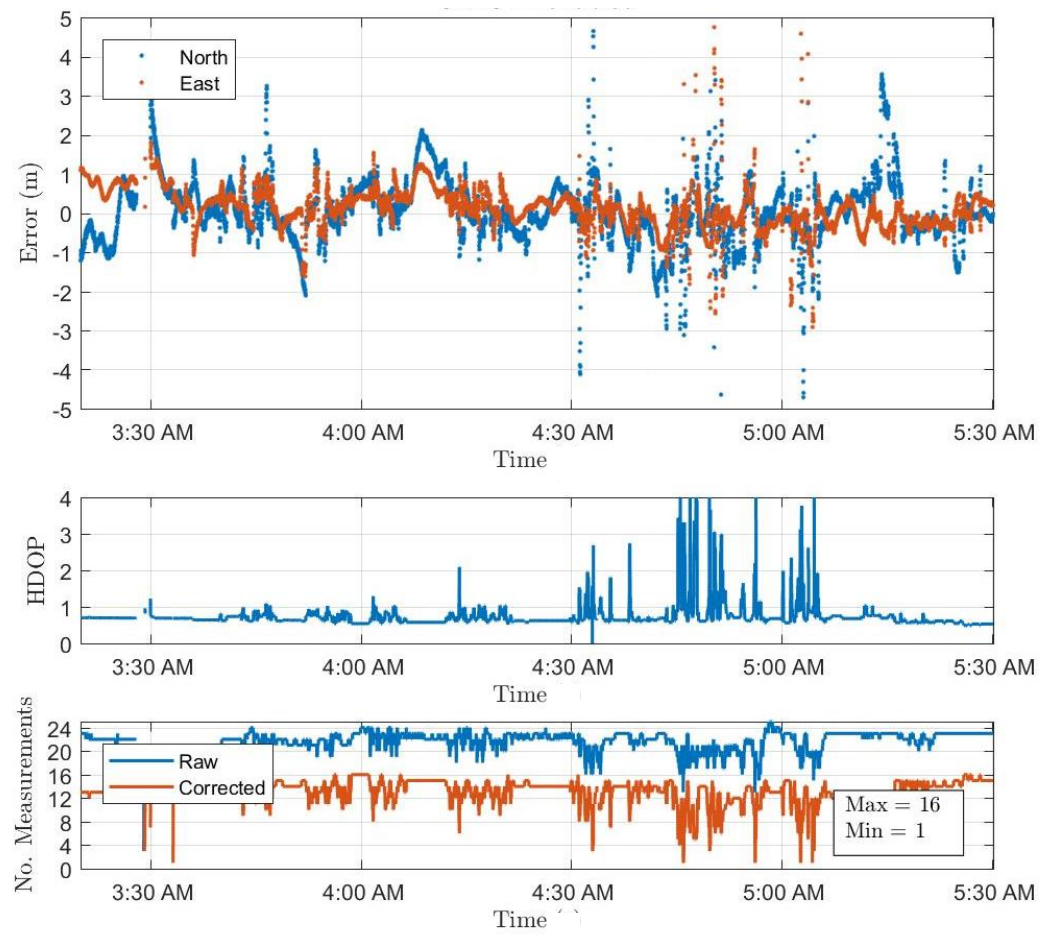

Figure 6: SBAS L1 results, suburban environment
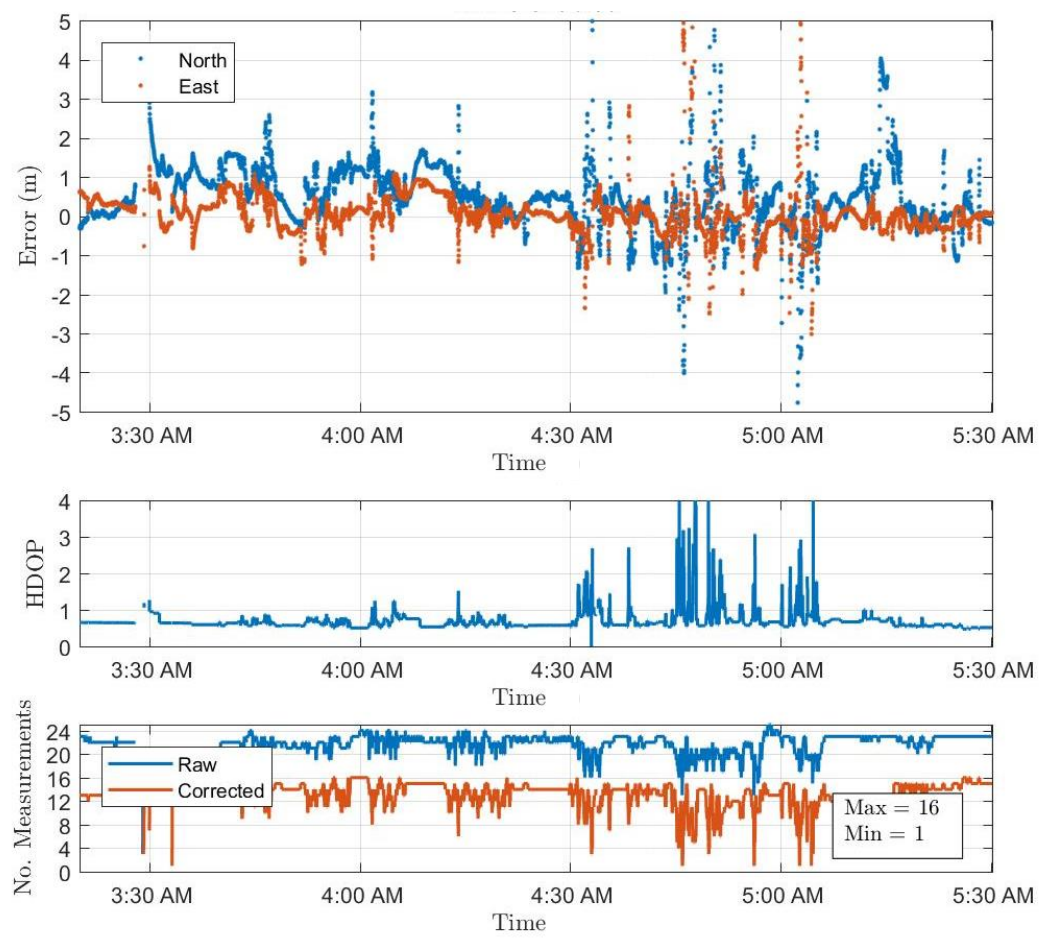

Figure 7: DFMC results, suburban environment 

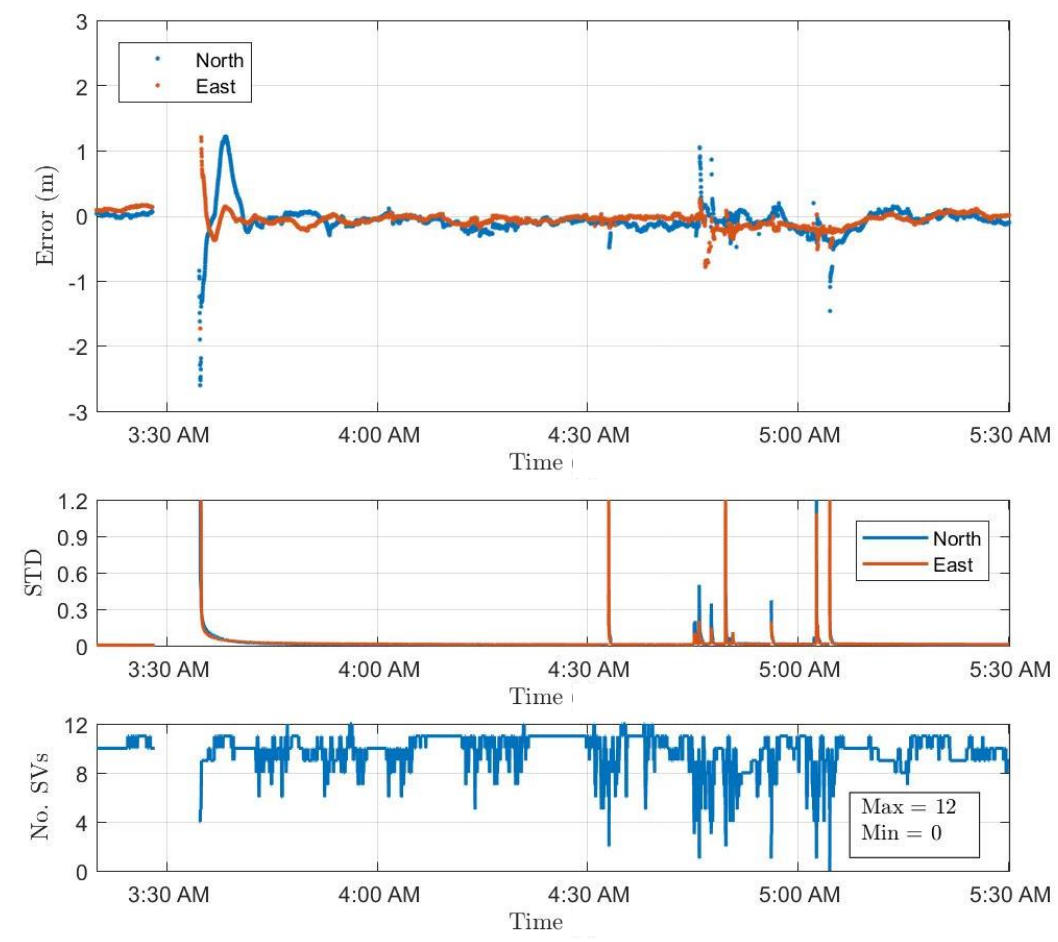

Figure 8: PPP results, suburban environment

In the Figures 6-8 (and in the upcoming similar Figures for other test cases), the HDOP of PPP results was not shown since they have a similar pattern to that of the DFMC-SBAS results, as both use dualfrequency ionosphere-free measurements, and the HDOP for DFMC is given. The standard deviations of the PPP were given to indicate the solution convergence behaviour. The standard deviations of the SPP and DFMC-SBAS are correlated to the shown HDOP and they are summarised in the Tables 2-6.

It can be seen from the Figures 6 to 8 that the positioning performance of both L1 and DFMC SBAS, and PPP is highly correlated with the number of satellites in view (see for instance the time stretch between 4:45AM and 5:15AM, GPS time). For PPP, the figures show the loss of visibility of some satellites (loss of lock) causing a temporary/partial loss of convergence, and some time was needed to reconverge to the previous precision levels.

Table 2 shows the average statistics for the full day of testing. Note that in this table and the Figures 6-8, as well as the coming similar tables and figures, SPP results are based on processing all available satellites, whereas L1 and DFMC SBAS used only satellites that have orbital and clock corrections. Likewise for PPP, only satellites with orbital and clock corrections were used. The table shows positioning availability, rate of outliers, mean error and RMSE. Figure 9 shows the mean absolute 2D horizontal error for the different positioning modes, which gives a good indicator of the system accuracy, irrespective of the sign of the error. The positioning results shown in this example are the best recorded among the different test runs. Similarly, Table 3 shows the average statistics for another test run in suburban environment in Sydney (Test Scheme 2).

The results experimentally demonstrate that both L1 and DFMC SBAS gave much better positioning accuracy than SPP, and they can provide sub-metre horizontal positioning accuracy for road applications when sufficient number of satellites can be observed. The tables also show that there is a significant difference in the mean error when comparing L1 SBAS with DFMC SBAS. This could be due to the first approach using interpolated values for the ionosphere delay (introducing some interpolation errors), while the latter removing the ionosphere delay, 
at the expense of increased observation noise, with the use of the ionosphere-free combination. This trade-off resulted sometimes in one method giving slightly better results than the other, and vice versa, depending on test conditions. However, the overall performance (in terms of availability, outliers' rate and accuracy) is close.

Table 2 - Statistics for testing in suburban environment in Wollongong

\begin{tabular}{|l|c|c|c|c|}
\hline \multicolumn{1}{|c|}{ KPI } & SPP & SBAS L1 & DFMC & PPP \\
\hline Availability & 0.96 & 0.99 & 0.99 & 1.00 \\
\hline Outliers rate & 0.04 & 0.03 & 0.04 & 0.02 \\
\hline Mean error North (m) & -0.90 & 0.00 & -0.19 & -0.05 \\
\hline Mean error East (m) & -0.08 & 0.15 & 0.04 & -0.03 \\
\hline Mean error Up (m) & -1.66 & -1.32 & -0.45 & -0.11 \\
\hline RMSE North (m) & 1.48 & 0.68 & 0.57 & 0.20 \\
\hline RMSE East (m) & 0.78 & 0.50 & 0.32 & 0.13 \\
\hline RMSE Up (m) & 2.69 & 1.75 & 1.24 & 0.39 \\
\hline
\end{tabular}

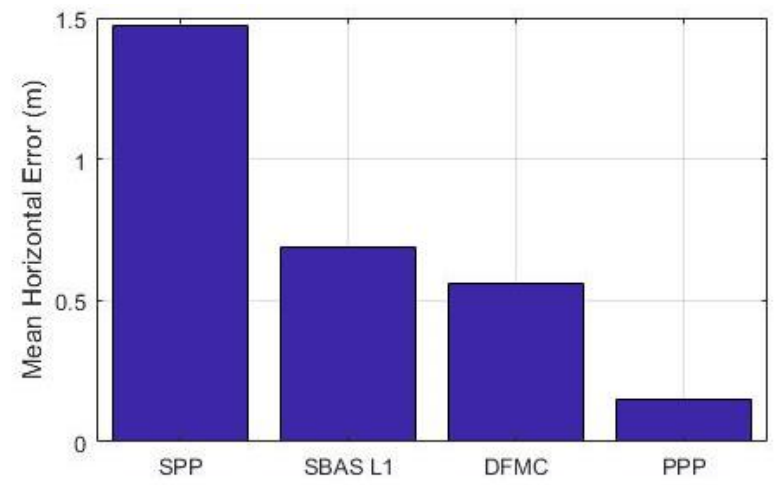

Figure 9 - Overall mean horizontal error for the test in Wollongong suburban environment

Table 3 - Statistics for testing in suburban environment in Sydney

\begin{tabular}{|l|c|c|c|c|}
\hline KPI & SPP & SBAS L1 & DFMC & PPP \\
\hline Availability & 0.94 & 0.99 & 0.99 & 1.00 \\
\hline Outliers rate & 0.04 & 0.03 & 0.03 & 0.02 \\
\hline Mean error North (m) & 1.18 & 0.40 & 0.17 & 0.16 \\
\hline Mean error East (m) & 0.72 & 0.47 & 0.29 & -0.14 \\
\hline Mean error Up (m) & 0.38 & -0.44 & 0.68 & 0.13 \\
\hline RMSE North (m) & 1.68 & 0.86 & 0.85 & 0.35 \\
\hline RMSE East (m) & 1.05 & 0.61 & 0.58 & 0.18 \\
\hline RMSE Up (m) & 2.89 & 1.56 & 1.90 & 0.28 \\
\hline
\end{tabular}

Naturally, PPP after convergence of the solution outperformed the L1 and DFMC SBAS methods in terms of all studied metrics, with accuracy at $0.2 \mathrm{~m}$. The appropriateness of the obtained performance and accuracy for lane level identification, which is the main objective of using SBAS positioning for ITS use, will be discussed in Section 5.6. 


\subsection{Results of low-density and high-density urban environments}

To show the performance of SBAS for land applications under variable satellite visibility conditions, a bus route was selected operating between Circular Quay to North Bondi in Sydney in Test Scheme 2, passing through different urban environments. The bus route was divided into three areas, i.e. high-density urban, low-density urban environments, as shown in Figure 4. For the low-density urban area, Figure 10 and Figure 11 show the time series of the positioning error, HDOP versus the number of observed satellites for DFMC SBAS and PPP. For the high-density urban area, the availability of positioning was below $30 \%$ for all positioning methods due to the presence of structure obstructing the signals. Accordingly, the graphs for this scenario are not shown due to the low number of measurements and positions available. The average positioning performance for the low-density and high-density urban areas is given in Table 4 and

Table 5, respectively.

Table 4 - Statistics for low-density urban environment in Sydney

\begin{tabular}{|l|c|c|c|c|}
\hline \multicolumn{1}{|c|}{ KPI } & SPP & SBAS L1 & DFMC & PPP \\
\hline Availability & 0.67 & 0.83 & 0.83 & 0.91 \\
\hline Outliers rate & 0.15 & 0.22 & 0.22 & 0.24 \\
\hline Mean error North (m) & 0.98 & 0.46 & 0.34 & 0.67 \\
\hline Mean error East (m) & 0.75 & 0.40 & 0.20 & -0.26 \\
\hline Mean error Up (m) & 0.43 & -0.68 & 0.60 & -0.06 \\
\hline RMSE North (m) & 2.38 & 1.71 & 1.75 & 0.99 \\
\hline RMSE East (m) & 1.42 & 1.17 & 0.98 & 0.56 \\
\hline RMSE Up (m) & 3.16 & 2.99 & 2.90 & 1.41 \\
\hline
\end{tabular}
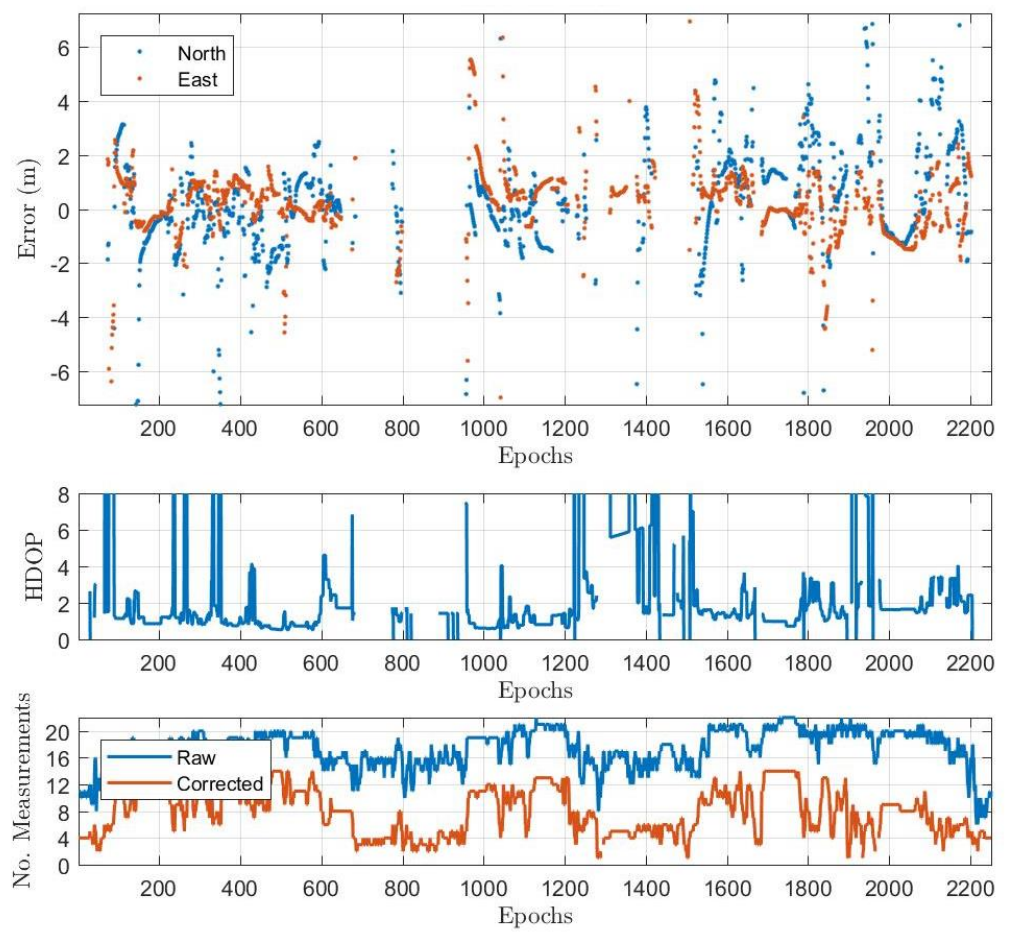

Figure 10: DFMC results, low-density urban environment, Test Scheme 2 (Sydney). 

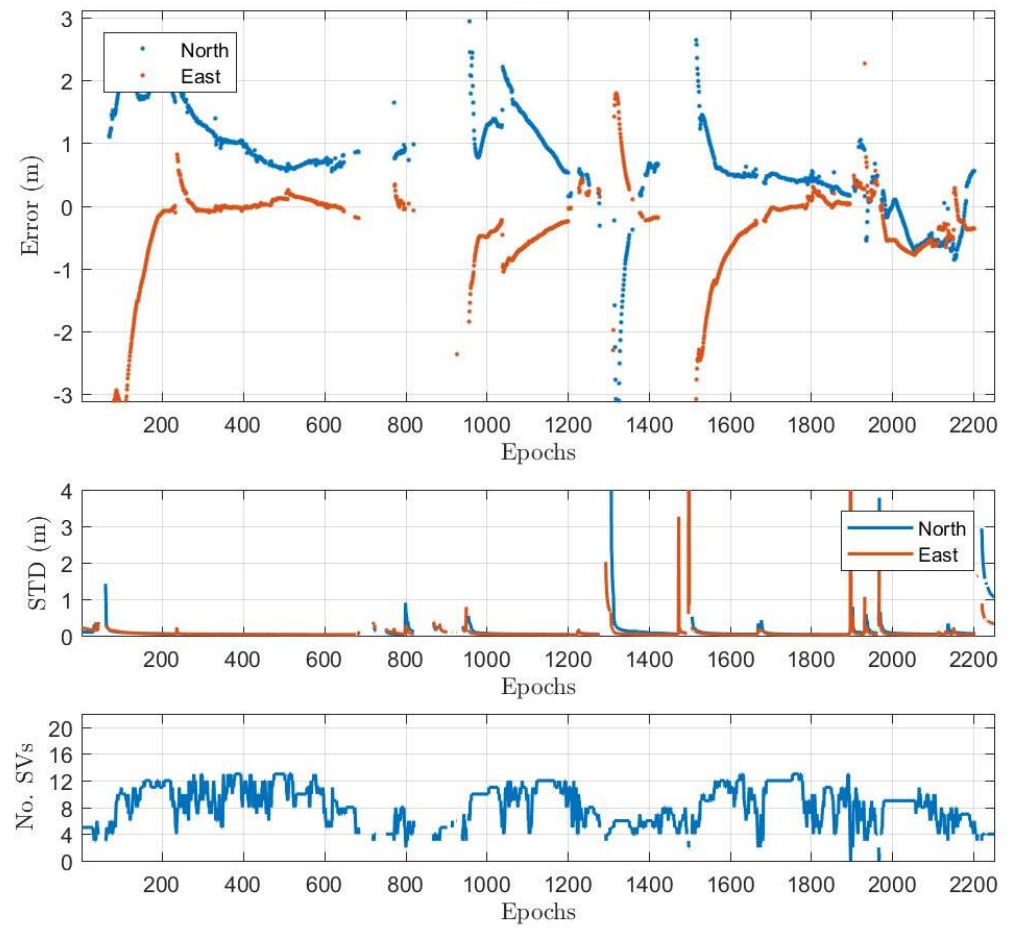

Figure 11: PPP results low-density urban environment, Test Scheme 2 (Sydney).

Table 5 - Statistics for high-density urban environment in Sydney

\begin{tabular}{|l|c|c|c|c|}
\hline \multicolumn{1}{|c|}{ KPI } & SPP & SBAS L1 & DFMC & PPP \\
\hline Availability & 0.11 & 0.29 & 0.29 & 0.21 \\
\hline Outliers rate & 0.10 & 0.15 & 0.10 & 0.15 \\
\hline Mean error North (m) & -0.60 & 2.90 & -0.22 & 0.23 \\
\hline Mean error East (m) & 1.22 & -0.19 & -1.73 & 0.51 \\
\hline Mean error Up (m) & -9.05 & -6.74 & -4.71 & 1.37 \\
\hline RMSE North (m) & 1.95 & 4.40 & 3.23 & 0.27 \\
\hline RMSE East (m) & 1.26 & 1.15 & 2.66 & 0.71 \\
\hline RMSE Up (m) & 9.08 & 8.01 & 5.68 & 1.65 \\
\hline
\end{tabular}

As the figures 10 and 11 show, the number of satellites in view in the urban environment was much lower on average than the suburban case, which affected both positioning accuracy and availability (with more scattered positioning results). For the PPP, the solution had frequently to re-initialise. Moreover, from the statistics in Table 4 and Table 5, it can be seen numerically how the performance of both SBAS and PPP methods is degraded by the urban environment. Availability of positioning is lower compared to suburban environment, due to unavailability of sufficient number of satellites to allow for positioning during several periods, which is a common problem for all GNSS positioning methods and not related to SBAS. The outliers' rate was also much higher. In this environment there is a slight improvement in terms of accuracy of the DFMC mode over the L1 SBAS, when available, for the reasons discussed earlier. 


\subsection{Results of the tree canopy environment}

Tree canopy is another environment that can significantly affect satellite visibility. For this case, a test was run in a hilly area of Wollongong (nearby Mount Ousley, Test 1.1 in Section 4), characterised by roads surrounded by bushes and trees. The presence of the mountain was further impairing the satellite visibility. Figure 12 illustrates the time series of the positioning error, HDOP and number of observed satellites in this test for the DFMC mode, given as an example, and the statistics of all positioning modes are given in Table 6 . As the figure shows, the tree canopy resulted in gaps in positioning for short periods and a significant degradation of positioning during most of the testing period.

As the satellite visibility varies continuously with time, the position errors were highly scattered. During testing, there was a stretch of clearer sky conditions between epochs 900 and 1600 , in which positioning error is more correlated (notice that SBAS modes use carrier phase smoothing), but it was not sufficient to improve the convergence of the PPP solutions. These parts of the figure show clearly the difference in performance between positioning in open sky versus that in tree canopy. The statistics given in the table 6 show that this environment causes a large number of outliers, however, the overall positioning performance was better when compared to the urban environment.
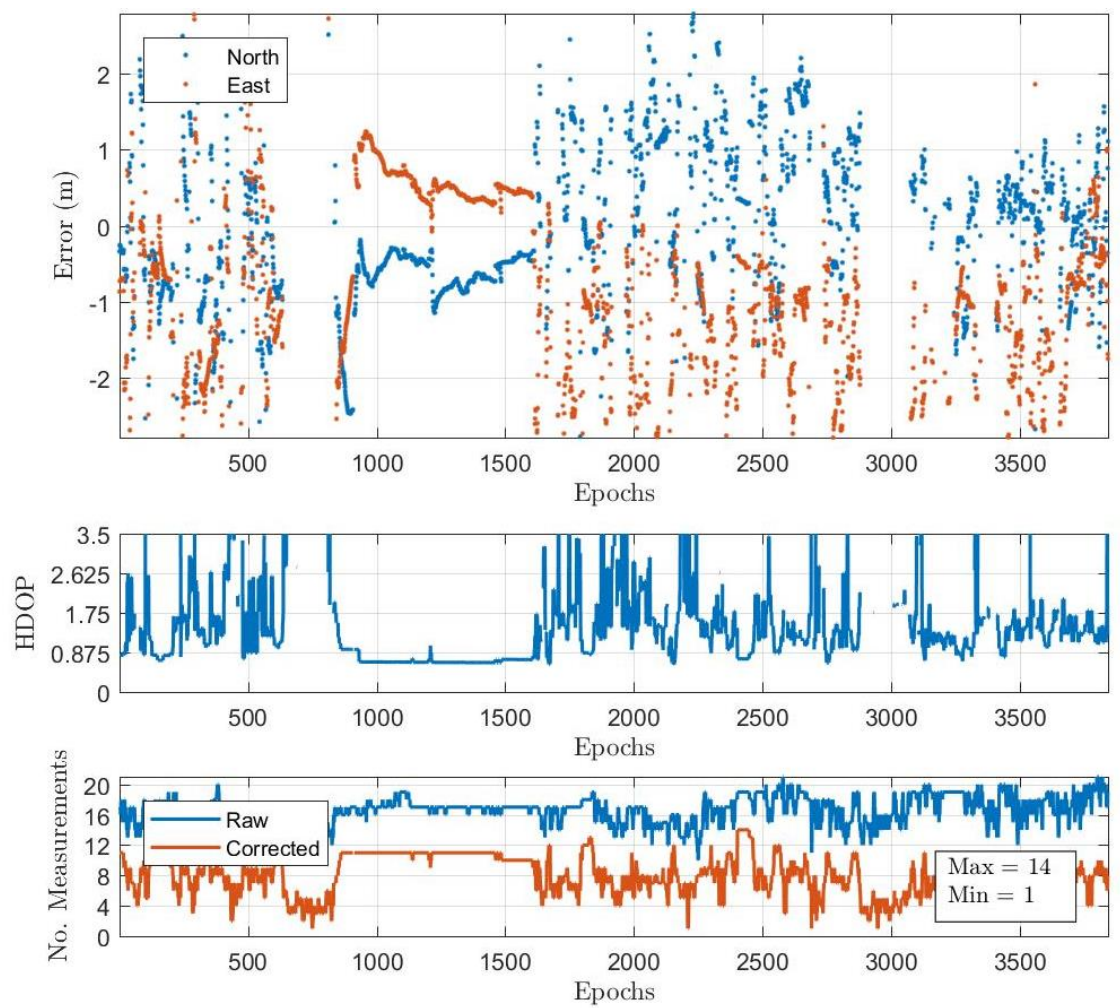

Figure 12: DFMC results, Test Scheme 1 (Wollongong), tree canopy

Table 6 - Statistics for tree environment in Wollongong when positioning was available

\begin{tabular}{|l|c|c|c|c|}
\hline \multicolumn{1}{|c|}{ KPI } & SPP & SBAS L1 & DFMC & PPP \\
\hline Availability & 0.51 & 0.87 & 0.87 & 0.97 \\
\hline Outliers rate & 0.06 & 0.16 & 0.18 & 0.26 \\
\hline Mean error North $(\mathrm{m})$ & -0.22 & -0.15 & -0.10 & -0.20 \\
\hline Mean error East $(\mathrm{m})$ & -0.66 & -0.66 & -0.92 & 0.16 \\
\hline
\end{tabular}




\begin{tabular}{|l|c|c|c|c|}
\hline Mean error Up (m) & 1.60 & -0.15 & -0.81 & -0.60 \\
\hline RMSE North (m) & 1.23 & 1.13 & 0.99 & 0.41 \\
\hline RMSE East $(\mathrm{m})$ & 1.09 & 1.29 & 1.68 & 0.35 \\
\hline RMSE Up (m) & 3.22 & 2.80 & 2.41 & 0.80 \\
\hline
\end{tabular}

\subsection{Analysis of Along-Track and Cross-Track positioning}

In transportation, it is also relevant to show positioning performance along the direction of travel of the vehicle (along-track, AT) in particular for collision avoidance and in the cross direction (cross-track, CT), which is of interest for lane identification applications. Figure 13 shows the horizontal errors for one test vehicle in a run of test 1.2 (Test Scheme 1, see Section 4) for DFMC results, shown as an example. In the top panel of the figure, the errors in the North-East reference frame are given, whereas at the bottom panel they are given in the vehiclefix Along-Track/Cross-Track reference frame.
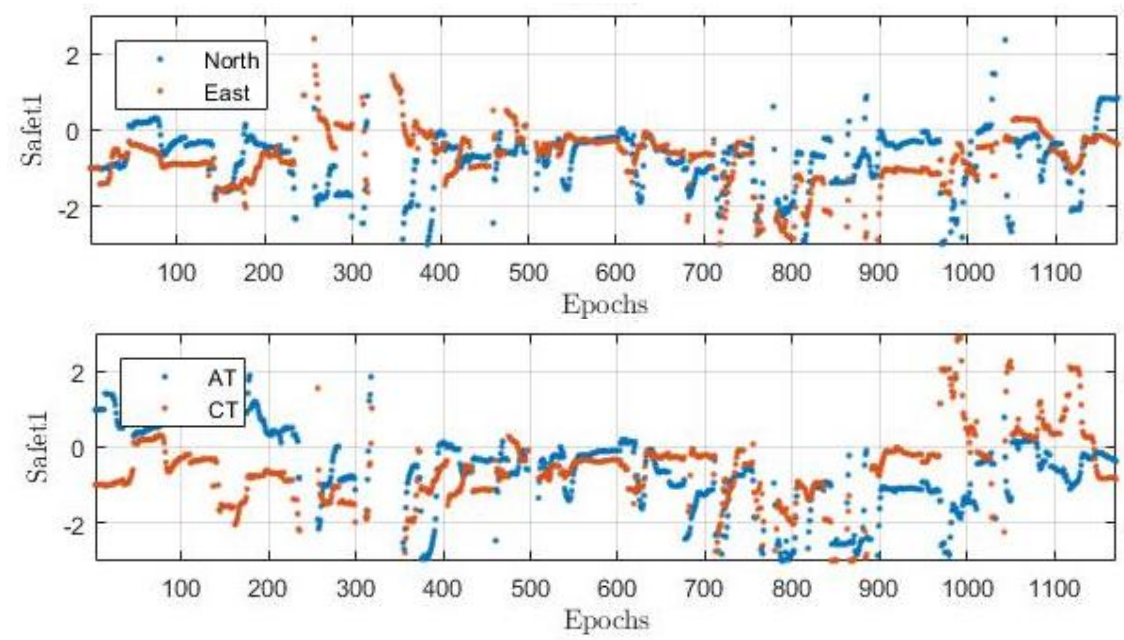

Figure 13: North-East (top) and corresponding Along-Track/Cross-Track (bottom) errors for one run

Results from this test and other test runs show that there were no significant differences between AT and CT errors, whereas there are noteworthy differences between errors in North and East directions - positioning in the East direction being 20\% to 50\% more accurate than in the North direction. This is a normal result in GNSS positioning in general in this region and relates to user location and geometry of satellites.

\subsection{Two vehicles results}

In transportation, as vehicles are traveling along with other vehicles, it is important not only consider the absolute positions of vehicles, but also their relative positions with respect to other surrounding vehicles sharing the road. Figure 14 shows, as a representative example, the horizontal errors (in North-East coordinates) from SBAS DFMC positioning in the top two panels of the figure for the two vehicles used in our tests (known as Safet1 and Tesla) during a run of test 1.2 (Test Scheme 1, see Section 4). The bottom panel shows the difference between the errors of the two vehicles.

From the two top rows one can notice a significant correlation between the two time series. This results in the difference between them having a smaller expected dispersion -comparable 
to the dispersion of each error time series at the top. This outcome can also be shown through the cross-correlation between the errors at the two vehicles, which was computed for different runs and was between 0.35 and 0.55 for both SBAS (L1 and DFMC) and PPP. The presence of a positive cross-correlation is desirable, as it could be exploited for relative positioning by Vehicle to Vehicle (V2V) communication.
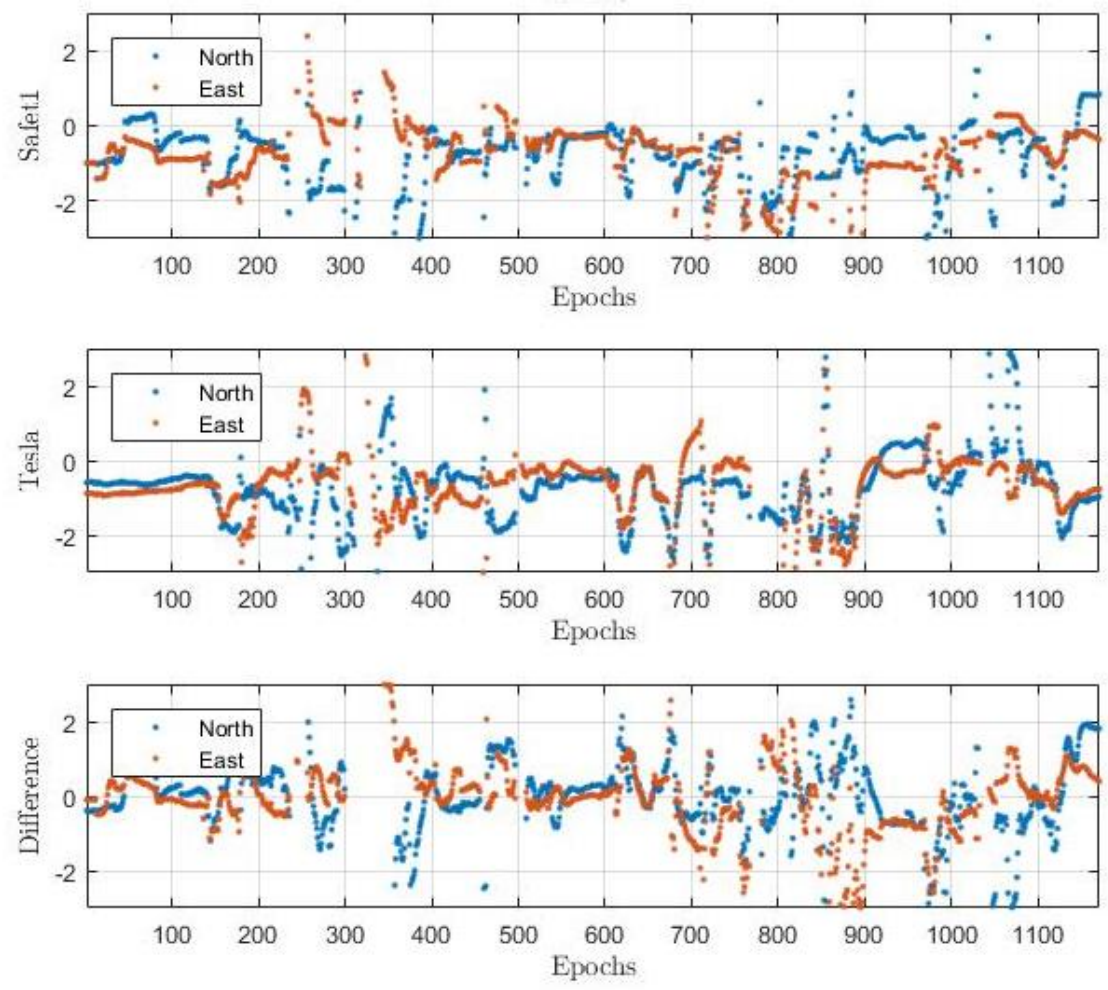

Figure 14: North-East errors in SBAS DFMC positioning for a run of test 1.2 (Test Scheme 1) for the two vehicles (top two panels) and their difference (bottom panel)

\subsection{SBAS for ITS applications}

The primary interest in this study is to investigate positioning using GNSS with SBAS for lane identification, i.e. identifying the lane where the vehicle is located in. The attention is therefore restricted to horizontal positioning. In Australia lane widths vary between $2.7 \mathrm{~m}$ (which is very rare) and $3.5 \mathrm{~m}$. Assuming a critical average lane width of $2.9 \mathrm{~m}$, and assuming that the GNSS antenna is located in the centre of the vehicle in the cross-direction, the rate of wrong lane identification is approximated by computing the percentage of epochs in which the Cross-Track positioning error of each vehicle is larger than 1.4 metres, i.e. less than half the lane width, such that the majority of the vehicle is in the lane. Figure 15 shows examples of successful lane identification during test 1.2 (Test Scheme 1, see section 4). The dashed lines represent the ground truth, which was obtained by relative kinematic positioning of the raw observations of the antenna of the vehicle collected by the SBAS receiver, processed with a nearby base station data in PPK. The continuous lines represent the positioning results obtained by SBAS.

The rate of false collision warnings was also estimated. Occurrence of a collision warning was linked to the estimated relative position between the two vehicles: when the estimated distance between the two vehicles exceeds a safety threshold, it is assumed a warning is raised. The J. of Spatial Science 2019 doi/full/10.1080/14498596.2019.166494316 
thresholds were selected at $4.5 \mathrm{~m}$ and $2.5 \mathrm{~m}$ for the Along-Track and Cross-Track components, respectively. For the scenario where a vehicle travelled on an overpass, another threshold of $2 \mathrm{~m}$ was further set for the relative height error. The values of the threshold were chosen based on the following assumptions:

- Average length of vehicles as 3.5m (allowing for an along-track separation of $1 \mathrm{~m}$ ).

- Average width of vehicles as $2 \mathrm{~m}$ (allowing for a cross-track separation of $0.5 \mathrm{~m}$ ).

- Average height of the vehicles as $1.5 \mathrm{~m}$ (allowing for a vertical separation of $0.5 \mathrm{~m}$ ).
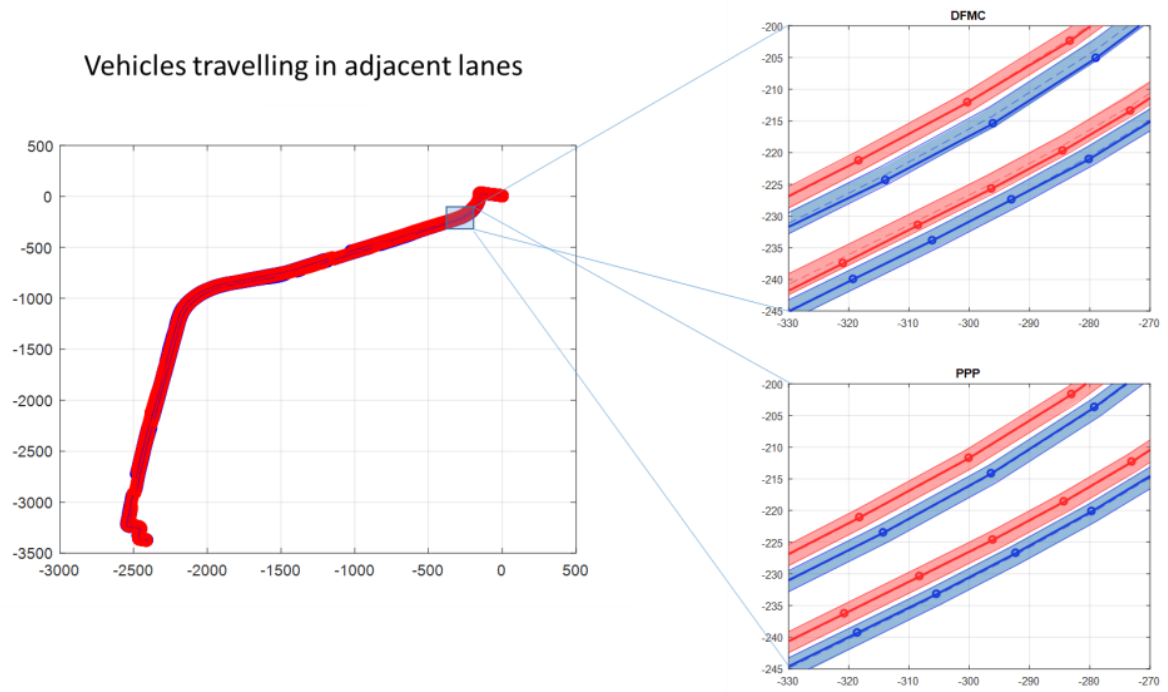

Figure 15: Run of test 1.2 (Test Scheme 1), examples of lane identification procedure in one section of one test. The dashed lines represent the ground truth, the continuous lines are the SBAS positioning results

Table to Table give a summary for the percentages of wrong lane identification for the two vehicles used in the tests (vehicle 1 is SAFET1 and vehicle 2 is Tesla), and the percentages of false alarm with the above pre-set thresholds for the SBAS L1, DFMC and PPP positioning in open sky environment. The results are shown for the following scenarios:

- Vehicles travelling in the same lane - Table 7 - (test scheme 1.1).

- Vehicles travelling in adjacent lanes in the same and opposite directions - Tables 8 and 9 - (test scheme 1.2).

- A vehicle at a cross street with the other vehicle approaching - Table 10 - (test scheme 2.1).

- A vehicle travelling on an overpass - Table 11 - (test scheme 2.2).

Table 7: Vehicles travelling in the same lane.

\begin{tabular}{|c|c|c|c|c|}
\hline KPI & Vehicle & SBAS L1 & DFMC & PPP \\
\hline \multirow{2}{*}{$\%$ wrong lane identification } & 1 & $14 \%$ & $21 \%$ & 0 \\
\cline { 2 - 5 } & 2 & $29 \%$ & $18 \%$ & 0 \\
\hline \% false alarms (forward collision) & $0.5 \%$ & $0.2 \%$ & $0.1 \%$ \\
\hline
\end{tabular}

Table 8: Vehicles travelling in adjacent lanes in the same direction.

\begin{tabular}{|c|c|c|c|c|}
\hline KPI & Vehicle & SBAS L1 & DFMC & PPP \\
\hline \multirow{2}{*}{$\%$ wrong lane identification } & 1 & $16 \%$ & $25 \%$ & $0.4 \%$ \\
\cline { 2 - 5 } & 2 & $17 \%$ & $22 \%$ & 0 \\
\hline \% false alarms (forward collision) & $5.1 \%$ & $2.8 \%$ & $0.1 \%$ \\
\hline
\end{tabular}


Table 7: Vehicles travelling in adjacent lanes in opposite directions.

\begin{tabular}{|c|c|c|c|c|}
\hline KPI & Vehicle & SBAS L1 & DFMC & PPP \\
\hline \multirow{2}{*}{$\%$ wrong lane identification } & 1 & $5 \%$ & $6 \%$ & 0 \\
\cline { 2 - 5 } & 2 & $34 \%$ & $20 \%$ & 0 \\
\hline \% false alarms (forward collision) & 0 & 0 & 0 \\
\hline
\end{tabular}

Error! Reference source not found. shows a sizeable difference in wrong identification rate between the two vehicles, possibly due to the positioning of vehicle 2 being affected by a temporary bias that caused a much larger number of lane identification mistakes. There was no difference in the equipment on-board the two vehicles, and it is believed that the difference in results is of random nature, as the trials were carried out for a short time span.

Table 10: A vehicle at a cross street with the other vehicle approaching.

\begin{tabular}{|c|c|c|c|c|}
\hline KPI & Vehicle & SBAS L1 & DFMC & PPP \\
\hline \multirow{2}{*}{$\%$ wrong lane identification } & 1 & $10 \%$ & $32 \%$ & 0 \\
\cline { 2 - 5 } & 2 & - & - & - \\
\hline \multicolumn{2}{|c|}{$\%$ false alarms (forward collision) } & 0 & 0 & 0 \\
\hline
\end{tabular}

Table 11: A vehicle travelling on an overpass.

\begin{tabular}{|c|c|c|c|c|}
\hline KPI & Vehicle & SBAS L1 & DFMC & PPP \\
\hline \multirow{2}{*}{$\%$ wrong lane identification } & 1 & $1 \%$ & $5 \%$ & 0 \\
\cline { 2 - 5 } & 2 & $9 \%$ & $6 \%$ & 0 \\
\hline \% false alarms (forward collision) & 0 & 0 & 0 \\
\hline
\end{tabular}

The results of the Table to Table indicate that in open sky environment, lane identification can be correctly performed $70 \%$ to $80 \%$ of the time with SBAS (either L1 or DFMC) and nearly all the time with PPP. False collision alarms, when defined with the small margins above, are quite rare, except for the scenarios with vehicles travelling in adjacent lanes, where they can reach $5 \%$ for SBAS modes (but still $<1 \%$ for PPP). In all other scenarios the false alarm rate was lower than $1 \%$. The float-ambiguity PPP solution as implemented in this study suffers from the long time needed for solution convergence. This can be acceptable for long routes such as highways, where signal interruptions and the need to re-initialise PPP are minimal. In addition, it is recommended that the use of PPP with ambiguity resolution (PPP-AR) be implemented in the future, supplemented by the ionosphere information computed in SBAS, since this method offers a shorter convergence time and possibly better accuracy (Nadarajah et al., 2018).

\section{SUMMARY AND DISCUSSION OF THE RESULTS}

Overall, due to the variable nature of the surrounding environment of the roads in transport applications, the results show significant difference in the positioning performance when moving among different environments including:

- The availability of SBAS and PPP, which varied from less than $30 \%$ to over $90 \%$.

- Accuracy ranged between $0.3 \mathrm{~m}$ and $3 \mathrm{~m}$.

This variation was attributed to urban and tree canopies, leading to the dividing results into four categories: suburban, low-density urban, high-density urban and tree canopy.

The main findings of testing the second generation L1 and DFMC SBAS with PPP capability can be summarised as: 
- Positioning performance of both SBAS and PPP methods is strongly dependent on the environment of application, as it is strictly linked to the strength of the satellite geometry, number of observed satellites, multipath and NLOS.

- The high-density urban environment was the least favourable condition with less than $30 \%$ availability for all correction types. Accuracy was in the order of $2 \mathrm{~m}$ to $3 \mathrm{~m}$ for SBAS with PPP was better at $1 \mathrm{~m}$ to $2 \mathrm{~m}$, where solution convergence was not reached. Moreover, the poor satellite availability led to corresponding high rate of outliers. Therefore it cannot be sole solution for transport related applications, and should be supplemented by other positioning sources such as Inertial Measurement units (IMU), cameras, radars and LiDAR.

- In less dense urban environments, the availability for the SBAS modes was over $85 \%$ and over $90 \%$ for PPP. Accuracy is in the order of $2 \mathrm{~m}$ along North and East directions for SBAS, $0.5 \mathrm{~m}$ to $1 \mathrm{~m}$ for PPP (highly varying in time due to losses of lock causing re-initialisation of convergence), and the rate of outliers was still high (>5\%) for all positioning modes.

- In suburban environments with good sky visibility, the availability of SBAS modes and PPP is over $98 \%$ and the outlier's rate was reasonably low. Accuracy was in the order of $1 \mathrm{~m}$ along North and East directions for SBAS and $0.1 \mathrm{~m}$ to $0.4 \mathrm{~m}$ for PPP.

- On road flanked by trees, creating a tree canopy (e.g. Mount Ousley in NSW), the performance was better than in urban environments, with availability over $85 \%$ for SBAS and $90 \%$ for PPP, giving accuracy of $1 \mathrm{~m}$ to $1.7 \mathrm{~m}$ for SBAS and $0.4 \mathrm{~m}$ to $0.5 \mathrm{~m}$ for PPP. However, the outliers' rate was high $(>10 \%)$.

- Positioning results from the DFMC mode slightly differ from the SBAS L1 mode mainly in terms of the mean error. However, overall accuracy and availability is close for the two modes. Our tests did not include periods of high fluxion of the ionosphere, where the use of DFMC might be more beneficial.

- When considering the suitability of these results to the tested ITS applications, results suggest that, in open sky environment, lane identification can be correctly performed $70 \%$ to $80 \%$ of the time with SBAS (either L1 or DFMC) and nearly all the time with PPP.

\section{CONCLUSION}

The results obtained during this study show that SBAS positioning data in open sky environment and with good satellite geometry can reach horizontal accuracy in the order of sub-metre. This level of accuracy is sufficient to place a vehicle within a lane on the transport network for use in ITS applications, something that is not possible to achieve reliably with existing GNSS SPP methods. This improved accuracy is important to the transport industry as it will aid ITS applications being developed in Australia and internationally. Since Australia now imports all vehicles from overseas, including countries and continents that have access to SBAS, Australian vehicle owners will be able to take advantage of SBAS-enabled hardware in the vehicles.

While accuracy of SBAS-based positioning in suburban environment can reach sub-metre accuracy, this accuracy deteriorates in high-density urban environments reaching a couple of metres, mainly due to presence of poor satellite geometry, multipath and NLOS. These are common concerns in positioning using GNSS that is shared with any other GNSS-based positioning technology. Therefore it cannot be sole positioning solution for transport related 
applications, and should be supplemented with other data sources such as IMU, cameras, radars and LiDAR. PPP is able to provide positioning with accuracy as low as 0.1 metre, however, it requires 30 to 60 minutes for solution convergence at start up or after visibility is lost for extended periods. This need for convergence makes the PPP solution less appealing for short journeys but it can be used for long trips, for instance along highways.

\section{ACKNOWLEDGEMENTS}

This work is funded through project PD8703 - SBAS for connected vehicles - part of the Australia/NZ SBAS Test-bed project. The authors would like to sincerely thank FrontierSI, Geoscience Australia (GA), and Land Information New Zealand (LINZ) for their support during the project. Acknowledgments are also due to the Transport for New South Wales and the University of New South Wales for their help in data collection. Davide Imparato helped in processing some of the data and its reporting.

\section{REFERENCES}

Ali, K., M. Pini, and F. Dovis, (2012), Measured performance of the application of EGNOS in the road traffic sector, GPS Solutions 16(2), 135-145.

Austroads (2015), Vehicle Positioning for C-ITS in Australia (Background Document), Technical Report, Project No. NT1632, Austroads Publication No. AP-R431-13, 2013.

Austroads (2015a), ITS Strategic Directions, Technical Report., Project No. NS1927, Austroads Publication No. AP-C95-15.

Blanch, J., T. Walter, P. Enge, Y. Lee, B. Pervan, M. Rippl, A. Spletter, and V. Kropp (2015), Baseline advanced RAIM user algorithm and possible improvements, IEEE Transactions on Aerospace and Electronic Systems 51(1), 713-732.

Choy, S., J. Kuckartz, A.G. Dempster, C. Rizos, \& M. Higgins (2017), GNSS satellite-based augmentation systems for Australia. GPS Solutions, 21(3), 835-848.

El-Mowafy, A., Deo, M. Kubo, N. (2017), Maintaining real-time precise point positioning during outages of orbit and clock corrections. GPS Solutions, 21(3), 937-947.

El-Mowafy, A. (2018). Real-Time Precise Point Positioning Using Orbit and Clock Corrections as Quasi-Observations for Improved Detection of Faults. J. of Navigation, 71(4), 769-787.

Enge, P., T. Walter, S. Pullen, C. Kee, Y-C. Chao, and Y-J. Tsai (1996), Wide Area Augmentation of the Global Positioning System, Proceedings of the IEEE 84(8), 1063-1088.

Gao, Y. and K. Chen (2004). Performance Analysis of Precise Point Positioning Using Real-Time Orbit and Clock Products, Journal of Global Positioning Systems 3, 95-100.

Heroux, P., Y. Gao, J. Kouba, F. Lahaye, Y. Mireault, P. Collins, K. Macleod, P. Tetreault, K. Chen (2004). Products and Applications for Precise Point Positioning - Moving Towards Real-Time, Proceedings of the ITM of the ION GNSS, Long Beach, CA, Sept $21-24,2004,1832-1843$.

Kechine, M., C. Tiberius, and H. Van der Marel (2003). Experimental verification of Internet-based Global Differential GPS, Proceedings of the ION GNSS, Portland, OR, Sept 9 - 12, 2003, 28 - 37.

Melgard, T., O. Orpen, J.H. Ulstein, and E. Vigen (2010). Pulling in All Signals, PPP with GPS and GLONASS: The New G2, GPS World March, 28-35.

Nadarajah, N., A. Khodabandeh, K. Wang, M. Choudhury and P.J.G. Teunissen (2018). Multi-GNSS PPP-RTK: From Large-to Small-Scale Networks, Sensors (Basel), 2018 Apr 3, 18(4):1078.

NSTB/WAAS T\&E Team (2015), Wide-Area Augmentation System performance analysis report, Technical report, October 2015, FAA/William J. Hughes Technical Center.

Roturier, B., E. Chatre, and J. Ventura-Traveset (2001), The SBAS integrity concept standardised by ICAO, Application to EGNOS, Navigation-Paris 49, 65-77. 
Tegedor, J., D. Lapucha, O. Ørpen, E. Vigen, T. Melgard, and R. Strandli (2015). The New G4 Service: Multi-constellation Precise Point Positioning Including GPS, GLONASS, Galileo and BeiDou, Proceedings of ION GNSS+, Tampa, FL, September 14 - 18, 2015, 1089-1095.

TIC (2012), Policy framework for Intelligent Transport Systems in Australia, Technical Report, Transport and Infrastructure Council, 2012.

Walter, T., J. Blanch, R. Eric Phelts, and P. Enge (2012), Evolving WAAS to serve L1/L5 users, Navigation 59(4), 317-327.

Zumberge, J.F., M.B. Heftin, D.C. Jefferson, M.M. Watkins, and F.H. Webb (1997). PPP for the efficient and robust analysis of GPS data from large networks, J Geo.Res.102, 5005-5017. 